\title{
UN ANÁLISIS SOBRE NUEVAS IMPLEMENTACIONES EN EL PROCESO DE EVALUACIÓN
}

\author{
Silvia Caronía (Universidad Nacional de Misiones)* \\ silvca2@gmail.com \\ Graciela C. Lombardo (Universidad Nacional de Misiones)** \\ gracielalombardo@gmail.com \\ Roxana Verónica Operuk *** \\ roxsoperuk@gmail.com
}

Recibido: 07/08/2012 Aceptado: 02/11/2012

\section{Resumen}

Este trabajo forma parte de un proyecto de investigación de carácter exploratorio y descriptivo. En él se presentan distintas cuestiones observadas en la implementación de recursos informáticos en diversas instancias del proceso de evaluación en la asignatura Geometría Proyectiva del Profesorado en Matemática de la FCEQyN. En particular se muestran la incidencia del uso del aula virtual y la utilización de softwares de presentación y específicos de la matemática en entrevistas clínicas grupales. Se toma a la evaluación como eje principal, en las primeras instancias del proceso (diagnóstico inicial y diagnóstico continuo).

La implementación de estos recursos informáticos permitió vislumbrar resultados satisfactorios, tanto en la información acerca del proceso de enseñanza y aprendizaje, como en el mejoramiento de las prácticas educativas.

\section{Palabras claves}

Recursos informáticos - Aula virtual - Entrevistas clínicas - Evaluación - Geometría.

* Especialista en Educación Superior. UNaM, Argentina. Profesora en Matemática, Física y Cosmografía FACENA-UNNE. Argentina.

** Magister en Educación en Ciencias con mención en Matemática. FI-UNCOMA, Argentina. Diplomada en Competencias TIC para docentes (Formación Alfaomega de Alfaomega Grupo Editor). Profesora en Matemática, Física y Cosmografía (FHyCS-UNaM, Argentina).

*** Profesora en Matemática, Física y Cosmografía (FHyCS-UNaM, Argentina). 


\section{Abstract}

This work is part of a research project of exploratory and descriptive. In this paper we show several issues identified in the implementation of information resources in various stages of evaluation, in the course Projective Geometry of the professoriate of Mathematics of FCEQyN. In particular, we show the impact of the use of virtual classroom and presentation software and mathematical software in the clinical interviews. The main axis is the evaluation and is taken in the first instances the process (initial diagnosis and ongoing).

The implementation of these computational allowed to glimpse satisfactory results in both the information about the process of teaching and learning, as in the improving of educational practices.

\section{Keywords} Geometry.

Computing resources - Virtual classroom - Clinical interviews - Evaluation -

\section{Introducción}

Este trabajo es un avance del Proyecto de Investigación "Análisis de la implementación de herramientas computacionales aplicadas al proceso de evaluación en Matemática" (1), que forma parte de una línea de investigación llevada a cabo por este grupo desde el año 2009: "Uso de la entrevista clínica para la evaluación continua en Geometría Proyectiva” (2) (Caronía, Lombardo, Operuk, 2009) y "Aplicación de herramientas metacognitivas integradas en el proceso de evaluación continua en la Geometría Proyectiva" (3), (Caronía, Lombardo, Operuk, Abildgaard, 2010-2011). En términos generales, los trabajos mencionados versan sobre el uso de herramientas metacognitivas para llevar a cabo la evaluación en todas sus dimensiones, en distintas promociones de la asignatura, Geometría III del Profesorado en Matemática de FCEQyN de la UNaM.

Como docentes de la Universidad, año a año hemos percibido la dificultad creciente que presentan los alumnos en el proceso de aprendizaje de los contenidos matemáticos del ciclo básico universitario. A pesar de que muchos de éstos son contenidos obligatorios de la currícula del nivel medio, los estudiantes no los reconocen como tratados en su escolaridad. De acuerdo a investigaciones realizadas (4), se ha detectado que, cuando los tiempos para desarrollar los contenidos matemáticos de la Currícula del Nivel medio escasean, hay contenidos del programa que "caen", algunos de ellos por ejemplo, inherentes a la Geometría.

En las investigaciones antes mencionadas se hace hincapié en dificultades observadas en: la resolución de problemas, decodificación del discurso del Profesor, lectura, escritura y debate sobre objetos matemáticos, capacidad de formular hipótesis, comprobarlas y elaborar argumentos sobre la validez de las mismas. Éstas, entre otras, son razones por las que los alumnos tienen 
grandes carencias conceptuales, lo cual repercute en el desempeño de sus actividades académicas. En particular, en la Geometría, ésta problemática se agrava aún más, porque se suman los inconvenientes, que tienen los estudiantes, en la construcción de objetos geométricos, interpretación de características y propiedades intrínsecas que esos objetos poseen, utilización de lenguaje específico, etc.

Al inicio del ciclo lectivo, los docentes nos enfrentamos con la incertidumbre de cuáles son los conocimientos que detentan los alumnos, si son suficientes para realizar un aprendizaje significativo de aquellos conceptos que abordarán en la Geometría. Por lo general, se acostumbra a someterlos a una "evaluación diagnóstica" la cual, en un gran número de casos, aporta información insuficiente en razón de ser un instrumento rígido, que no sólo no tiene en cuenta la forma en que están entramados los conocimientos adquiridos en años anteriores, sino tampoco la singularidad psíquica del alumno. Además, al finalizar cada núcleo temático se implementan pruebas parciales, que forman parte de la acreditación de la asignatura.

Se piensa que no sólo es primordial integrar al alumno como actor fundamental en el proceso de enseñanza y aprendizaje, sino también considerar a la evaluación como vía potente que posibilita al docente hacer una retroalimentación de las actividades realizadas en su quehacer. Se considera imprescindible encontrar otros instrumentos evaluativos, que permitan determinar cuáles son los conocimientos previos de los estudiantes, cuáles están relacionados con la temática a enseñar, cuáles están incorporados en forma significativa en su matriz cognoscitiva, como así también la forma en que se interrelacionan.

Ahora bien, por todo lo manifestado precedentemente, percibimos la necesidad de pensar una nueva manera de formación. Partimos del supuesto que la implementación de nuevas tecnologías de la información y la comunicación, podrían propender a una enseñanza y aprendizaje diferentes. En tal sentido, encarar la investigación en forma paralela, sería necesario para estudiar el efecto alcanzado, en nuestro caso, en la geometría.

En esta etapa investigativa, dirigimos la mirada hacia materias del Profesorado en Matemática, apoyados en la teoría de la Asimilación de David Ausubel, y con el objetivo de mejorar la enseñanza de la Geometría Métrica y Geometría Proyectiva, asignaturas del $1^{\circ}$ y $3^{\circ}$ año* respectivamente.

En este trabajo se presenta algunas cuestiones observadas en la implementación de los recursos informáticos en instancias del proceso de evaluación en Matemática, en particular la incidencia del uso del aula virtual en la "evaluación diagnóstica inicial", la utilización de softwares de presentación y específicos

\footnotetext{
Del Plan de Estudios de la Carrera del Profesorado en Matemática, de la Facultad de Ciencias Exactas, Químicas y Naturales (FCEQyN) de la Universidad Nacional de Misiones. (UNaM).
} 
de la matemática, en entrevistas clínicas grupales "evaluación diagnóstica continua", en la asignatura Geometría Proyectiva.

En tal sentido, existen antecedentes relevantes como el de Rodríguez Conde (2011), quien, en su trabajo, expone la importancia de la aplicación de las TIC a la evaluación de alumnos universitarios, aseverando que la tecnología puede ser una herramienta útil en los aspectos más mecánicos del proceso de evaluación, y que es necesaria la existencia de criterios pedagógicos explícitos que guíen su aplicabilidad; en tanto que Huerta (1998) utiliza, en su investigación, la entrevista clínica, como herramienta complementaria de los mapas conceptuales confeccionados por alumnos, herramientas que le otorgaron información potente acerca de los aprendizajes obtenidos.

\section{Marco teórico}

El fundamento teórico que sustenta esta estrategia de evaluación, se basa en la corriente epistemológica del Constructivismo, el cual se nutre de las teorías de Piaget, Vygotski, Ausubel, entre otros. A diferencia de otras corrientes, esta centra su atención en el sujeto cognoscente, quien puede entenderse como un producto de su conocimiento, como consecuencia de lo que extrae del medio desde la interacción social en consonancia con parámetros sociales, y todo el bagaje cognoscitivo interno, el cual es realizado y modificado constantemente en todo instante de su vida.

Para Ausubel la nueva información será potencialmente aprendida y retenida siempre y cuando los conceptos relevantes sean claros y estén disponibles en la estructura cognitiva del individuo. Si los conceptos tienen la virtud de oficiar de nexos para la nueva información, ésta adquirirá significado y pasará a ser un elemento estable y relevante y es así que se produce el aprendizaje significativo. Todo concepto que ha sido incorporado significativamente en la estructura cognitiva es cimiento para el asiento de la nueva información, la que potencialmente tendrá significado para el individuo. Este proceso se producirá en forma sucesiva cada vez que ingrese nueva información, y en la medida que se vaya eslabonando con ideas o conceptos relevantes previos, lo cual implica que al producirse un nuevo aprendizaje significativo, consecuentemente se reestructurará la organización jerárquica conceptual hasta entonces existente.

\section{Proceso de evaluación}

En toda la actividad docente queda de manifiesto el tipo de práctica evaluativa, la concepción de enseñanza y de aprendizaje que el docente posee y realiza. En el proceso evaluativo no solamente se evalúan los conocimientos que el alumno ha adquirido, sino también de qué forma lo hace, cómo se contribuye para alcanzar esa meta, la efectividad del diagnóstico continuo realizado para seleccionar los contenidos en función del grupo presente, y también la 
auto-evaluación docente que resignifica todos los resultados obtenidos durante $y$ al final del proceso.

En el ámbito académico, usualmente suele decirse que la evaluación es llevada a cabo mediante un proceso, pero casi siempre el docente concluye realizando el control y registro de los resultados obtenidos por los alumnos. Según Palou de Maté (2003), existen dentro del aula tres instancias fundamentales en el proceso de evaluación, las cuales se complementan mutuamente, por el sentido que cada una de ellas tiene. La autora denomina a esas instancias: Diagnóstico Inicial, Evaluación Diagnóstica Continua y Acreditación.

En la primera etapa (Diagnóstico Inicial) la finalidad es establecer cuáles son los saberes alcanzados por los alumnos en los años anteriores; en la siguiente (Evaluación Diagnóstica Continua) determinar cuáles son los nuevos conocimientos adquiridos a fin de enmarcar la propuesta de enseñanza, como también establecer criterios de valoración de los aprendizajes, y en la última fase (Acreditación) el propósito está centrado en la verificación de resultados para certificar y legitimar sus conocimientos ante la Institución.

En acuerdo con Carlino (2005), la evaluación, en este sentido, representa un medio potente en el que se produce la retroalimentación del aprendizaje y de la enseñanza. En efecto, lo producido por el alumno en instancias evaluativas confiere información al docente la cual puede ser devuelta al alumno, a fin de reorientar su desempeño, como así también proporcionar argumentos para repensar su práctica pedagógica posterior. Además, "la función tácita de toda evaluación: señalar a los alumnos qué es importante en una materia". (Carlino, 2005: 107)

\section{Entrevista clínica}

La entrevista clínica tiene sus antecedentes en trabajos de psicoanálisis realizados en el siglo XIX, posteriormente fue perfeccionada por Jean Piaget en la década de los veinte del siglo pasado. Mediante su uso, Piaget evaluaba la capacidad cognitiva de los niños y adolescentes. "La teoría de Piaget, junto con los métodos de entrevistas asociados a ella para registrar datos sobre cómo razonan los niños, condujo a la identificación de cuatro estadios de desarrollo..." (Novak y Gowin, 1988, p. 146). Posteriormente, Joseph D. Novak, las utilizó con los fines de determinar los conocimientos previos de los alumnos y la evolución producida durante el proceso de instrucción. Esta evolución está referida al concepto de aprendizaje significativo introducido por David Ausubel.

Las entrevistas clínicas son herramientas potentes de gran utilidad en la investigación educativa. Su aplicación permite determinar no solo las estructuras cognoscitivas de los educandos, sino también preconceptos y concepciones erróneas que puedan poseer y de muy difícil desarraigo. 
En otras palabras, se deben determinar cuáles son los conceptos más importantes (centrales) relacionados con el material a enseñar que se encuentran firmemente establecidos en la memoria de largo plazo, como así también la forma en que están relacionados entre ellos. Estos conceptos tienen la particularidad de ser claros y estables para el estudiante, siendo por lo tanto los más pertinentes (en términos de la teoría de la asimilación) para actuar como subsumsores del nuevo material, o, lo que es lo mismo, funcionar como elementos de "anclaje" donde la nueva información que se va a impartir quedará firmemente "encadenada". (Chrobak, 1998: 11).

En la aplicación de estas herramientas metacognitivas, se recomienda su planificación, es decir, diseñar con antelación las preguntas a formular, prever el lugar y momento donde se llevarán a cabo, como también respetar un tiempo de duración que no exceda los 30 minutos. Los resultados obtenidos de los registros en las entrevistas dependerán del grado de flexibilidad de las preguntas realizadas: a mayor nivel de flexibilidad mayor es la posibilidad de determinar los conocimientos y competencias que poseen los alumnos, como así también estrategias de aprendizaje, formas de razonamiento, etc. De acuerdo al momento de la instrucción, en el que sea implementada, servirá para el diagnóstico inicial o para el diagnóstico continuo, lo cual permite al docente evaluar los conocimientos previos, los adquiridos durante el proceso de enseñanza aprendizaje y, además, reflexionar y repensar acerca de su propia práctica docente.

De acuerdo a los autores Johnson, Johnson, Holubec, (2004) no hay parámetros ideales para la elección del número de integrantes de un grupo. Existen diferentes variables que indican la dimensión más conveniente, por ejemplo objetivos propuestos, edades, experiencia en trabajos de esta índole, tipo de material didáctico y/o bibliográfico a utilizar, tiempo disponible para la actividad, entre otras. La diversidad de destrezas, competencias y habilidades generalmente está en proporción directa al número de integrantes, el mayor número de éstos implica destinar mayor tiempo para que cada uno exponga su punto de vista y a su vez conjugarlo con los restantes.

\section{Metodología}

El presente trabajo es de caracter exploratorio y descriptivo y se enmarca dentro del Paradigma Hermenéutico o interpretativo del campo socio educativo.

Apoyados en la teoría de la Asimilación de David Ausubel, se toma como referencia a una parte de la población del Profesorado en Matemática en la materia Geometría Proyectiva del $3^{\circ}$ año, asignatura dictada en el $2^{\circ}$ cuatrimestre del ciclo lectivo 2011. 
Como instrumentos de análisis se utilizaron los registros provenientes de: cuestionarios para los alumnos respondidos a través del aula virtual y correspondieron a la primera instancia de evaluación diagnóstica inicial, y luego las entrevistas clínicas grupales en las que se utilizaron softwares de presentación y softwares matemáticos, correspondiendo ésta a la evaluación continua.

Esta información, de las primeras instancias de la evaluación, constituyó el medio de reflexión del docente para delinear y perfilar su actividad docente.

\section{Análisis de avances}

Se presenta, a modo de ejemplo, una parte de los resultados parciales de la Investigación. Más precisamente la implementación de las acciones correspondientes al curso de Geometría Proyectiva.

\section{Diagnóstico inicial}

El primer día de clases del año 2011, se solicitó a los alumnos que en forma individual ingresen al aula virtual de la cátedra a fin de responder un cuestionario que se había subido especialmente para esa oportunidad. Para luego postear, en el mismo sitio, las respectivas respuestas. Asimismo, se explicaron los motivos y alcance de la actividad, que servirían como aportes positivos a la enseñanza, aprendizaje y fundamentalmente contribuirían a tomar conocimiento de los saberes previos relativos a los conceptos que se abordaran en la asignatura.

Los propósitos que se establecieron para implementar la toma del cuestionario individual fueron:

a) Determinar conocimientos previos de los alumnos relativos a sistemas axiomáticos en la matemática en general y en la Geometría clásica en particular.

b) Establecer la visión que tienen los alumnos respecto del cumplimiento de las propiedades de la Geometría euclidiana ante la presencia de transformaciones como la proyección y sección.

En el Cuadro 1 se muestra el criterio de valoración elegido de acuerdo a la respuesta dada por el educando a la pregunta que se le formulara. Este criterio permitió hacer un registro global de de los conocimientos previos que detentaban los alumnos cursantes. 
Cuadro 1. Criterio de valoración de las respuestas en función del nivel de conceptualización

\begin{tabular}{|c|c|c|c|c|}
\hline VALORACIÓN & $\mathbf{0}$ & $\mathbf{1}$ & $\mathbf{2}$ & $\mathbf{3}$ \\
\hline CRITERIO DE & No & $\begin{array}{c}\text { Presencia de } \\
\text { preconceptos o } \\
\text { Conceptos erróneos } \\
\text { o espontáneos en } \\
\text { la respuesta dada }\end{array}$ & $\begin{array}{c}\text { Responde a } \\
\text { la pregunta } \\
\text { en forma } \\
\text { mediana- } \\
\text { mente } \\
\text { adecuada }\end{array}$ & $\begin{array}{c}\text { Responde a } \\
\text { la pregunta } \\
\text { en forma } \\
\text { adecuada }\end{array}$ \\
\hline
\end{tabular}

Las preguntas que se formularon se organizaron en dos grupos, las cuales constan en los Cuadro 2 y Cuadro 3 respectivamente.

Cuadro 2. Primer grupo de preguntas del cuestionario

Respecto a términos indefinidos y sistemas axiomáticos

1) ¿Qué es un término indefinido o concepto primitivo?

2) ¿Podrías dar ejemplos de términos indefinidos?

3) ¿Qué es un axioma o postulado?

4) Da al menos un ejemplo de axioma.

5) ¿Cuál es la diferencia entre axioma y teorema?

6) Da al menos un ejemplo de sistema axiomático

7) ¿Depende el sistema axiomático de los términos indefinidos que se utilicen para formularlo? Explica.

En relación a las preguntas del Cuadro 2, algunos alumnos aludieron no recordar qué eran términos indefinidos, otros dieron una definición incorrecta: "es algo que no puede ser demostrado", y un número reducido contestó correctamente A pesar de esta dificultad, hubo quienes pudieron consignar ejemplos válidos.

En general, respondieron correctamente al respecto del significado de axiomas como también la diferencia existente con los teoremas.

Un escaso número de alumnos supieron dar ejemplos correctos de sistemas axiomáticos como también la dependencia existente entre el conjunto de términos indefinidos y el sistema axiomático creado a partir de éstos. 
Cuadro 3. Segundo grupo de preguntas del cuestionario

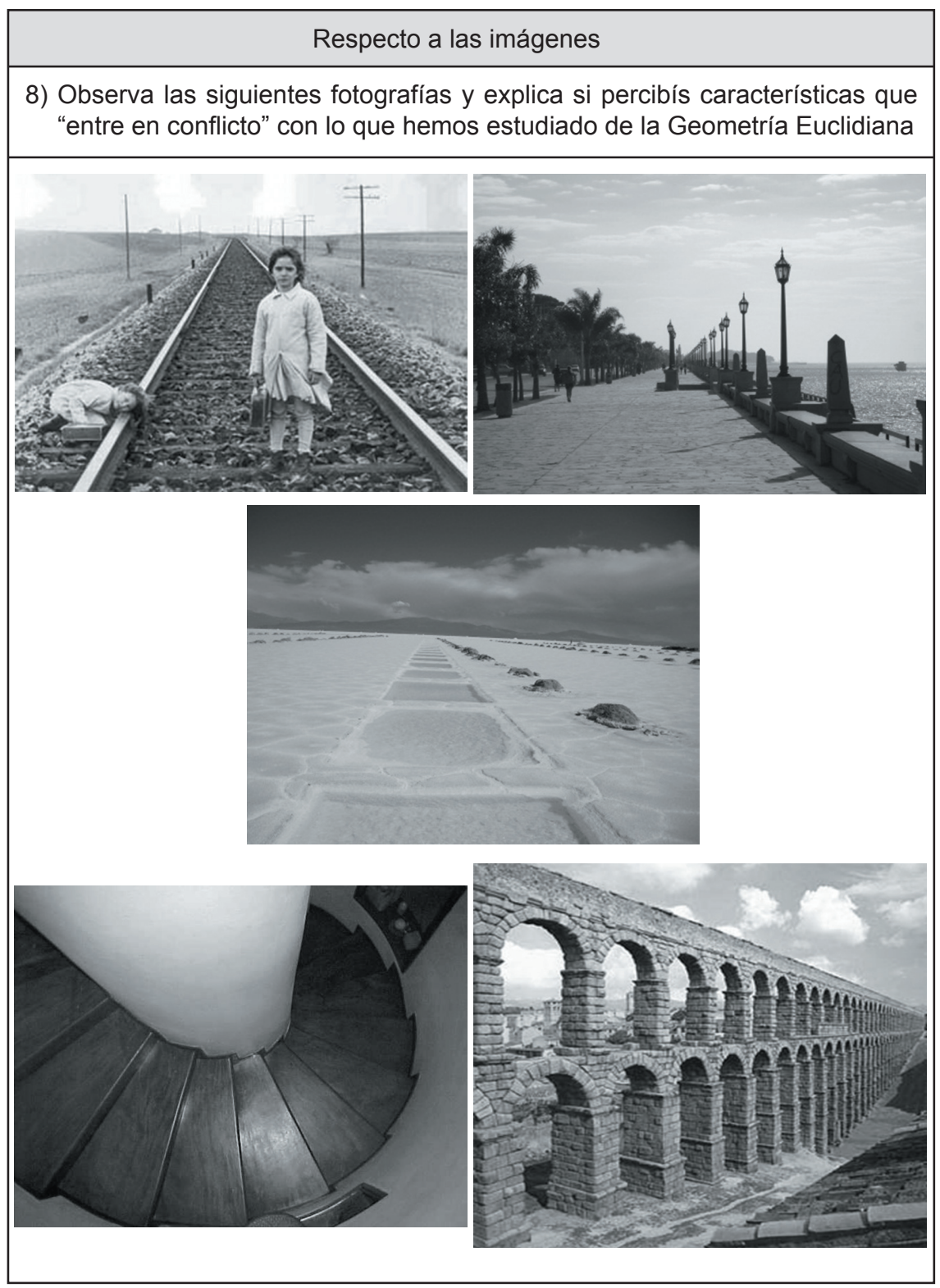




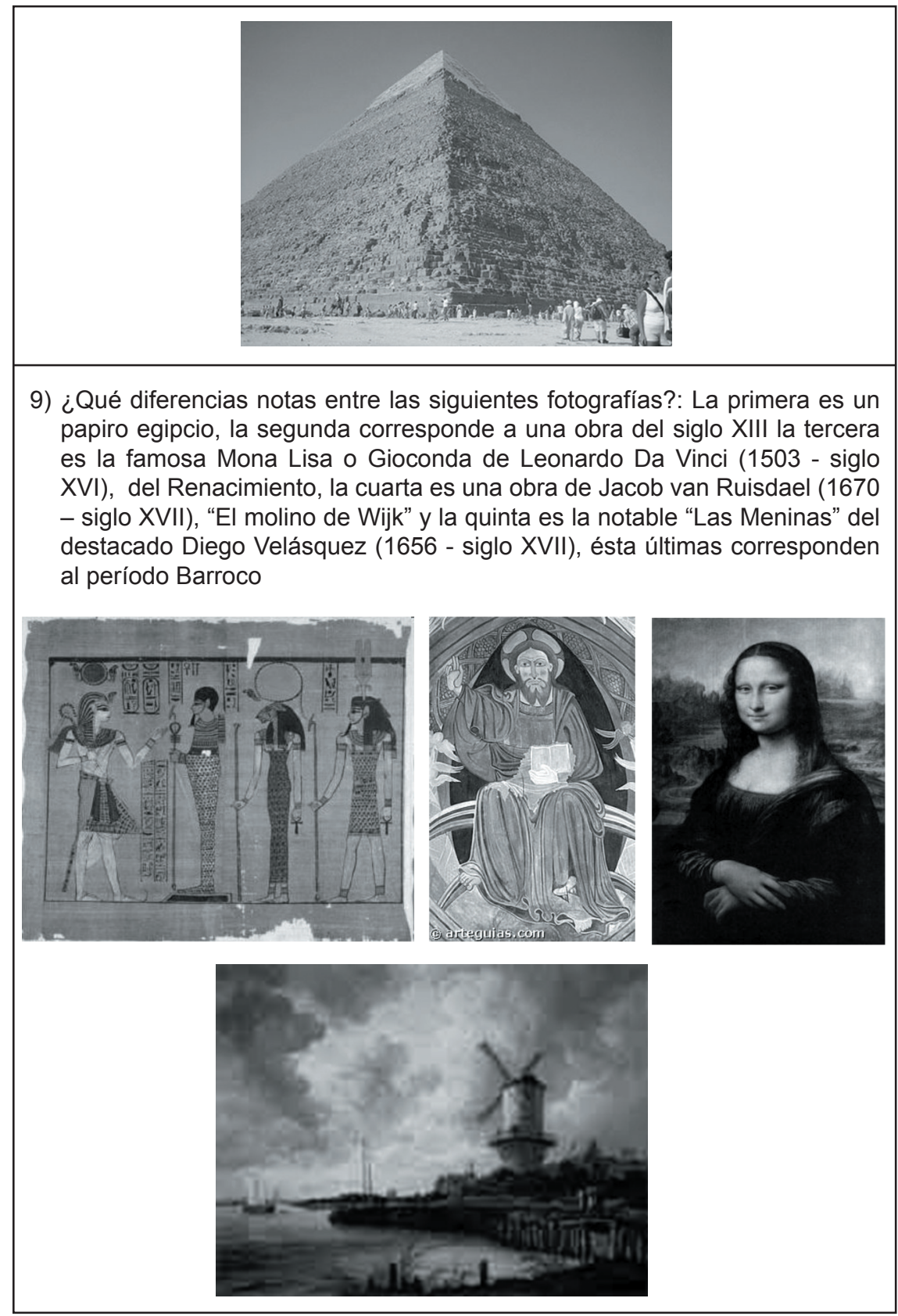




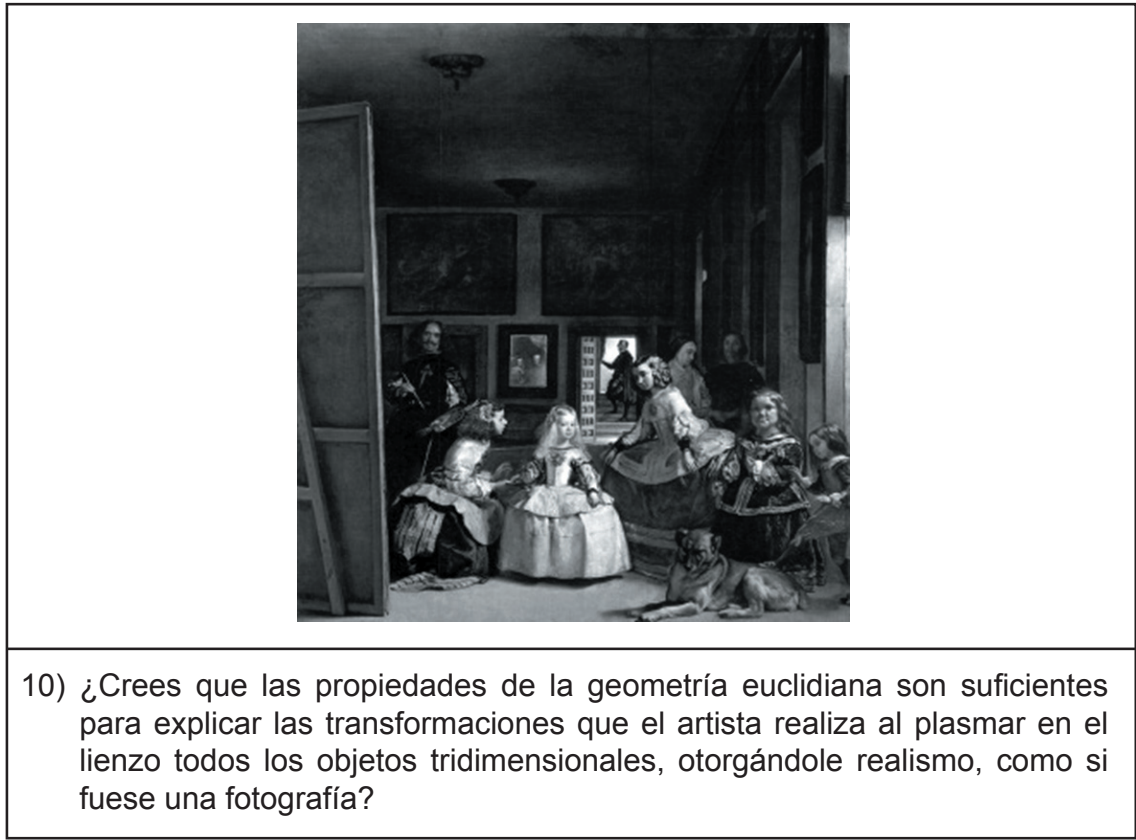

Pudo detectarse presencia de concepciones alternativas en respuestas dadas a la primera pregunta del Cuadro 3: hubo quienes aludieron que la Geometría euclidiana solo estudia la geometría del plano y no la del espacio, es decir consideraban a esta última como una Geometría no euclidiana.

Si bien, en la generalidad de las respuestas dadas a la primera pregunta del Cuadro 3, los alumnos pudieron establecer que en las fotografías presentadas no se cumplía el paralelismo de líneas rectas, conservación de longitudes y de dimensiones, congruencia de figuras, equivalencia de superficies. Solo un par de alumnos pudieron percibir la no conservación de la amplitud de ángulos en la transformación producida por el fenómeno de la fotografía. Aunque la conservación de ángulos está íntimamente ligada a la conservación de longitudes y áreas, el hecho que los alumnos no lo hayan explicitado, hizo presuponer que probablemente no lo observaran como una característica a destacar.

Haciendo un análisis comparativo e integral entre los resultados obtenidos de las respuestas dadas por los alumnos, un mayor número de ellos fueron capaces de hacer un análisis crítico y adecuado a partir de la observación de las fotografías y las imágenes de obras pictóricas, hasta entonces novedoso para ellos.

En cambio en menor número tuvieron presente los conceptos abordados en los años previos, tales como términos indefinidos, axiomas, sistemas 
axiomáticos, teoremas, etc. Esto mostraría que los aprendizajes sobre esos temas no fueron significativos, pues de ser así, deberían verse reflejados en las respuestas dadas.

Esta instancia, dentro del proceso de evaluación en general, posibilitó recabar información potente para rever la metodología a aplicar, enmarcar la propuesta de enseñanza, establecer criterios de valoración de los aprendizajes y determinar la existencia de concepciones alternativas, a efectos de revertirlas.

\section{Entrevistas clínicas grupales}

Al inicio del ciclo lectivo se acordó, con los alumnos, la constitución de equipos reducidos, conformado por tres integrantes como máximo. Estos grupos tuvieron la tarea de llevar a cabo trabajos de estudio sobre temas de la Geometría Proyectiva (no desarrollados aún en las clases), para luego, en una fecha acordada, defender sus producciones, dar muestra de un genuino trabajo colaborativo, ya que no solo serían evaluados por el desempeño individual sino por la interacción entre los miembros del equipo.

Los objetivos establecidos para implementar las entrevistas clínicas grupales fueron:

a) Lograr que los alumnos aborden temas inéditos como un proceso de construcción del conocimiento.

b) Propiciar que los alumnos realicen análisis y discusiones sobre la temática presentada, a través de la negociación de significados en un ambiente de camaradería y cooperación.

c) Realizar una evaluación integrada que tenga en cuenta, por un lado los aprendizajes individuales de los alumnos y por otro, el cumplimiento del objetivo del trabajo en equipo como propiciador de los aprendizajes de todos sus integrantes. (Evaluación diagnóstica continua)

d) Evaluar cuáles son los conocimientos que ofrecieron dificultad en el aprendizaje, o en los que no se produjo aprendizaje alguno, y determinar una estrategia para revertir esas situaciones, como medio apropiado para evaluar la práctica docente. (Evaluación diagnóstica continua)

e) Propender el uso de recursos informáticos como una instancia complementaria para su formación.

Durante el cursado de la asignatura se realizaron en dos oportunidades entrevistas grupales, las que se describirán en cada una de ellas, al igual que los resultados obtenidos en cada una de ellas. Si bien las preguntas se diseñaron con antelación a las entrevistas, se hicieron algunas modificaciones en función de las respuestas dadas por los alumnos entrevistados, procurando promover en ellos procesos asociativos y/o reflexivos conforme lo ameritaba la situación.

En relación a esta instancia de la investigación se realizó un análisis de las entrevistas clínicas grupales, tomando el mismo criterio de valoración para las 
entrevistas clínicas individuales (Cuadro 1). A diferencia de aquel, se adicionó una línea destinada a observaciones referidas al desempeño del equipo en cuanto al nivel de cohesión logrado como facilitador del aprendizaje de todos sus integrantes.

\subsection{Primera entrevista clínica grupal}

Los propósitos que se establecieron para implementar la primera entrevista grupal fueron:

a) Comprobar que los alumnos adquirieron el significado de homología en general y de las homologías particulares, y la capacidad de establecer relaciones con los conceptos correspondientes abordados en la Geometría I. (Evaluación diagnóstica inicial y evaluación diagnóstica continua)

b) Analizar el grado de conceptualización logrado por los alumnos en torno a las cónicas como homológicas de la circunferencia, métodos constructivos sustentados por la proposición de Desargues. (Evaluación diagnóstica continua)

Las preguntas formuladas se consignan en el Cuadro 4.

Con la formulación de las tres primeras preguntas, del Cuadro 4 , se determinaron el nivel de conceptualización alcanzado en torno a los conceptos que subyacen en la proposición de Desargues, tales como eje y centro de homología, puntos homólogos y rectas límites. Esta instancia fue propicia para realizar la evaluación diagnóstica de los aprendizajes de conceptos trabajados en clases anteriores, cimientos para el abordaje de los nuevos temas objetos de estudio que realizaron los jóvenes.

En cuanto a la cuarta pregunta, del Cuadro 4, se establecieron que los educandos disponían, como elementos de anclaje, conceptos tales como posiciones relativas de una circunferencia y una recta. La posesión de estos conceptos no es una cuestión menor, por ser un tema que probablemente tengan que enseñarlo en su futura labor docente.

En tanto, la quinta y sexta perfilaron la relación existente entre los elementos de la recta límite de un sistema y sus respectivos homólogos en el otro. Para poder obtener las diferentes variedades fue necesario que los jóvenes realicen precisas construcciones tomando como fundamento a la proposición de Desargues, explícitamente requerido en la última pregunta. 
Cuadro 4. Preguntas formuladas en la primera entrevista clínica grupal

\begin{tabular}{|l|}
\hline \multicolumn{1}{|c|}{ Respecto a las Homologías particulares } \\
\hline 1) ¿Cuáles son las homologías particulares que pueden enumerar? \\
\hline 2) ¿Cómo se caracterizan cada una de ellas? \\
\hline $\begin{array}{l}\text { 3) ¿Observaron diferencias en la denominación que da el autor respecto a las } \\
\text { conocidas por ustedes? }\end{array}$ \\
\hline \multicolumn{1}{c|}{ Respecto a las cónicas } \\
\hline $\begin{array}{l}\text { 4) ¿Cuáles son las diferentes posiciones relativas que pueden tener una } \\
\text { podrán, en cada caso, tener las mismas? }\end{array}$ \\
\hline $\begin{array}{l}\text { 5) ¿Cuáles son los homólogos de los puntos de intersección considerados en } \\
\text { cada uno de los casos? ¿Qué inferencias realizaron respecto de estos puntos } \\
\text {-los homólogos- en relación a la cónica a la que pertenecen? }\end{array}$ \\
\hline $\begin{array}{l}\text { 6) A partir de conocer la posición relativa de una circunferencia y la recta límite del } \\
\text { mismo sistema, ¿cuáles son las cónicas que obtuvieron como homológicas de } \\
\text { la circunferencia? ¿Me pueden explicar en cada caso el método constructivo } \\
\text { utilizado? }\end{array}$ \\
\hline
\end{tabular}

\subsection{Segunda entrevista clínica grupal} fueron:

Los objetivos propuestos para implementar la segunda entrevista grupal

a) Comprobar que los alumnos logren apropiarse de los conceptos de cónica envolvente y cónica lugar, y de establecer relaciones con las correspondientes nociones de perspectividad y proyectividad abordadas previamente. (Evaluación diagnóstico continuo)

b) Analizar el grado de conceptualización e inferencia alcanzado por los alumnos en torno a las diferentes variedades de cónicas y los elementos de tangencia en cada uno de los casos. (Evaluación diagnóstico continuo) En el Cuadro 5 se citan las preguntas que se formularon en la oportunidad. Con la formulación de la primera pregunta se indagó acerca de la interpretación hecha por los jóvenes en cuanto a los entes geométricos engendrados en cada caso. Con la segunda y tercera se estableció si habían inferido que, en el caso de ser proyectivos, los haces de rectas y las puntuales, entonces la cónica obtenida degenera en dos rectas y dos haces de rectas respectivamente.

En esta instancia no solo se valoraron los aprendizajes producidos durante el trabajo de estudio, sino también la relación que los educandos establecieron con los conceptos vistos en clases previas. 
Cuadro 5. Preguntas formuladas en la segunda entrevista clínica grupal

Cónica envolvente y cónica puntual - Métodos constructivos

1) ¿Podrían definir cónica envolvente y cónica lugar?

2) ¿Por qué en la definición que han dado de cónica envolvente aludieron a puntuales proyectivas y no perspectivas, qué ente se obtendría si las puntuales fueran perspectivas?

3) ¿Por qué en la definición que han dado de cónica lugar aludieron a haces proyectivos y no perspectivos, qué ente se obtendría si los haces fueran perspectivos?

4) ¿Cómo se clasifican cada una de ellas? Justifiquen en cada caso.

5) ¿Cuáles son los elementos de tangencia en la cónica envolvente? Y ¿Cuáles son los una cónica lugar? ¿Cómo se determinan?

Al requerir, en la cuarta pregunta, la clasificación de las cónicas envolvente y lugar, se observaron que de acuerdo a las particularidades que puedan tener los pares de elementos correspondientes en los espacios generadores respectivos, los alumnos infirieron que en cada uno de los casos pueden obtenerse elipses, parábolas e hipérbolas. En esta instancia se evaluó cómo los jóvenes relacionaron los conceptos previos, en pos de la generación de nuevos conocimientos (evaluación diagnóstica continua e inicial).

Con la formulación de la última pregunta se escudriñó acerca de las inferencias producidas respecto de los elementos de tangencia en las cónicas, teniendo en cuenta que éstos resultan ser duales ya que las cónicas envolvente y lugar también lo son. Cabe destacar, que éste no es un análisis sencillo de realizar, pero se pretendió que los alumnos pudieran efectuarlo durante el trabajo de estudio grupal. En algunas entrevistas, se percibió que los estudiantes no habían logrado tal cometido, es por ello que se reformuló la pregunta para que emerja la conclusión pretendida.

\subsection{Uso de Softwares}

En las distintas oportunidades que se implementaron las entrevistas clínicas, los alumnos expusieron y explicaron sus producciones mediante algún software de presentación y el GeoGebra, los cuales les permitieron dar fundamento al trabajo de estudio realizado.

Cabe señalar que el software GeoGebra fue utilizado como un recurso adicional tanto en el desarrollo de las clases como en la toma de exámenes parciales. Si bien una mayoría de los alumnos conocía su uso, en la primera clase práctica, se hizo una presentación y explicación en forma general. 


\subsection{Ejemplos de las entrevistas clínicas grupales}

A continuación se muestran algunos ejemplos de trabajos realizados cuando los grupos hicieron sus defensas mediante un software de presentación (Figura 1).En todos los casos utilizaron los recursos que éstos disponían, como ser: animaciones, efectos de transición, links a construcciones en GeoGebra (Figura 2).

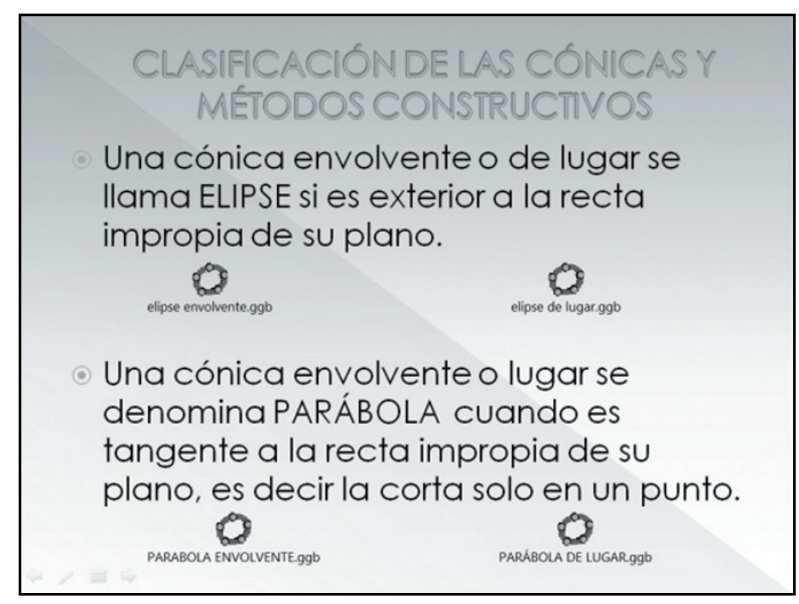

Figura 1. Diapositiva de presentación con links a construcciones en GeoGebra

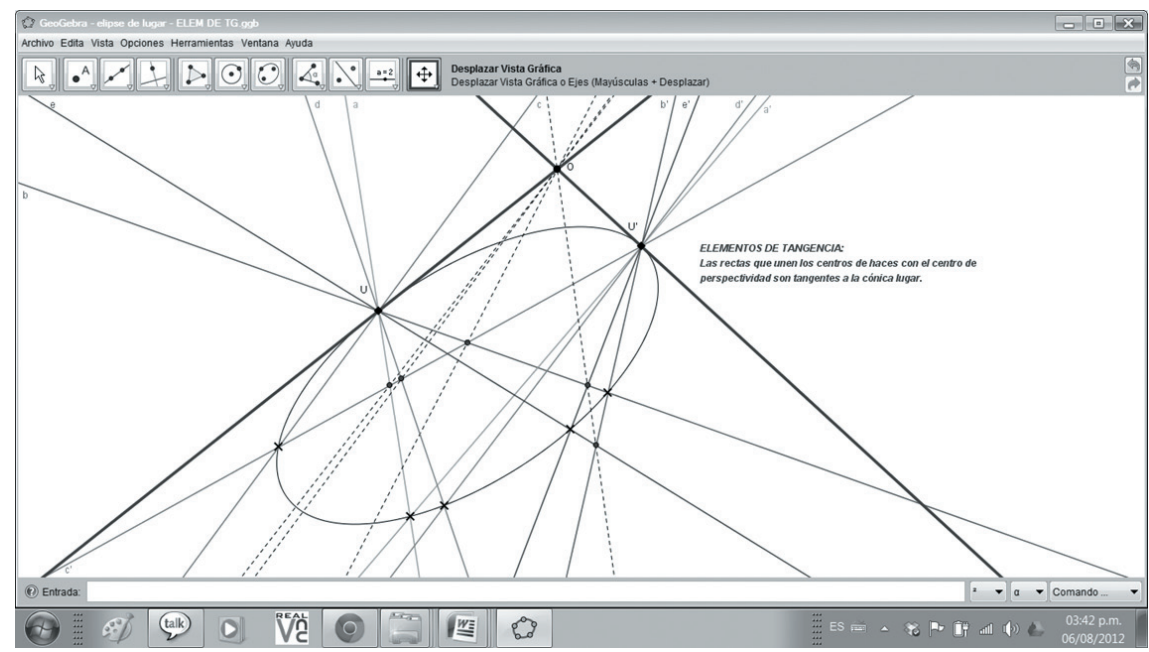

Figura 2. Producción en GeoGebra presentada por un grupo de alumnos 
En cuanto a estas construcciones, las realizaron en forma correcta recurriendo al "paso a paso" (Figura 3) para recrearlas, y en algunos casos mostraron el "protocolo de construcción" (Figura 4), lo cual evidenció no solo experticia en su uso, sino también la posibilidad de utilizar el software como medio de evaluación de las acciones realizadas.

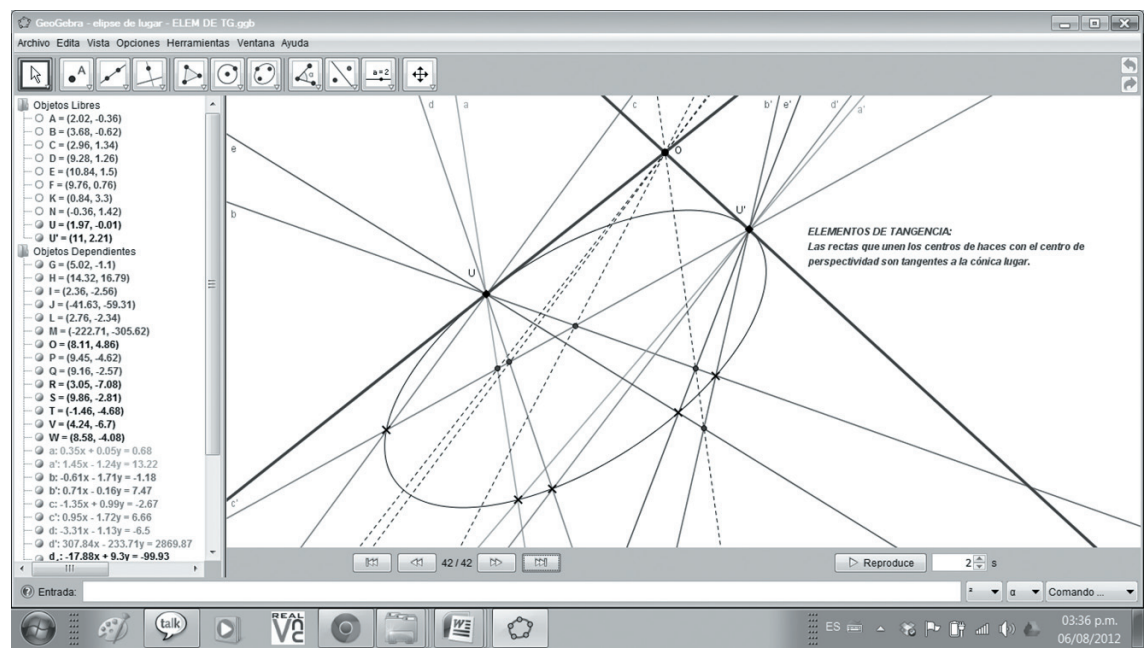

Figura 3. Producción en GeoGebra, con el uso del paso a paso.

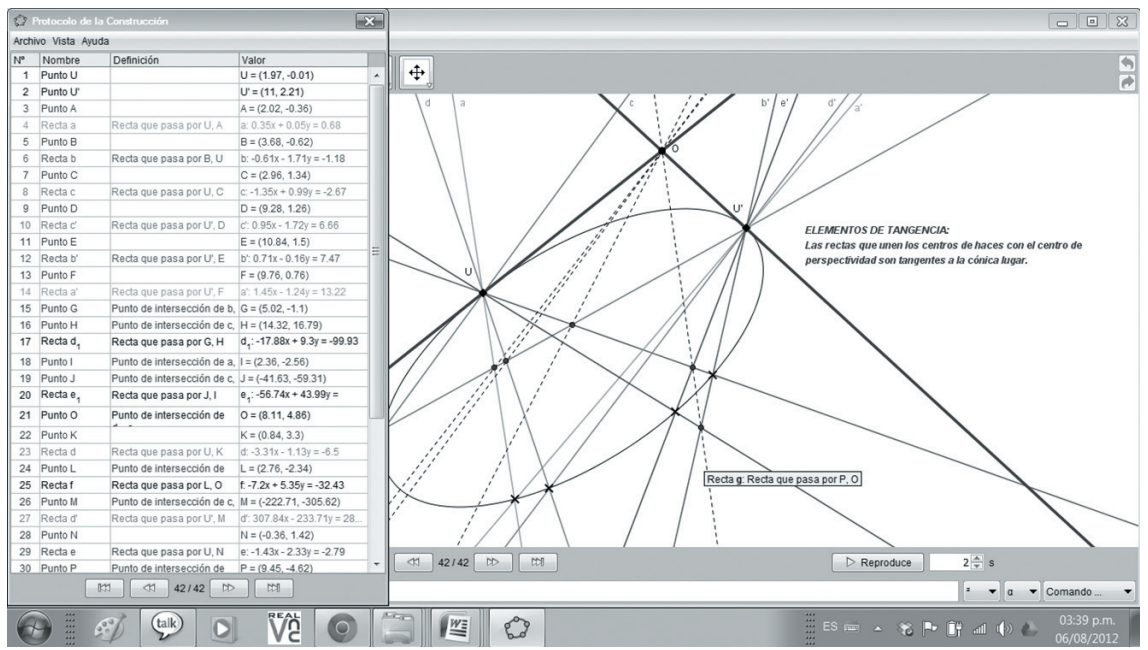

Figura 4. Producción en GeoGebra, con protocolo de construcción 
En general, los estudiantes usaron un adecuado léxico específico; tuvieron en cuenta las recomendaciones hechas respecto al trabajo en equipo como propiciador de construcción de conocimientos, negociación de significados, aprendizajes y metas de comprensión y, tal como fuera anticipado, reconocieron que estas son instancias adecuadas para concretar aprendizajes complementarios y realizar auto evaluaciones.

\section{Conclusiones}

Tal como se observa, esta investigación, hasta la fecha muestra que:

Con la entrevista clínica individual se pudo obtener información eficaz para rever la metodología a aplicar, enmarcar la propuesta de enseñanza, establecer criterios de valoración de los aprendizajes logrados, determinar la existencia de concepciones alternativas a fin de revertirlas y propender a la autoevaluación de los alumnos.

La formalización de las entrevistas clínicas grupales se constituyeron en instancias propicias para llevar a cabo la evaluación diagnóstico inicial y continua.

Las actividades grupales, realizadas por los estudiantes, fueron acciones apropiadas a efectos de propender la integración entre pares, las cuales permitieron el intercambio de opiniones y puntos de vista, promovieron el proceso de comprensión y consecuentemente el aprendizaje significativo.

De acuerdo a este primer análisis comenzamos a vislumbrar resultados satisfactorios en beneficio de sus actores: los alumnos reconocieron a estas instancias como adecuadas para concretar aprendizajes complementarios y realizar auto evaluaciones; y los docentes como medio satisfactorio para continuar con este método de evaluación.

\section{Notas Bibliográficas}

(1) Proyecto de investigación incentivado (Código 16Q500).

(2) Trabajo de investigación independiente, aprobado según Resolución CD № 265-11.

(3) Proyecto de investigación incentivado (Código 16Q476), aprobado según Resolución CD No 182-11.

(4) Caronía, Zoppi, Operuk y otros (2005-2008). "Los conocimientos matemáticos en el umbral de la Universidad: una asignatura pendiente" Proyecto de investigación incentivado (Código 16Q288).

\section{Referencias Bibliográficas}

- $\quad$ Burdón (2005) El espíritu de la Colmena. Recuperado el 01 de agosto de 2006, del sitio web de Escritura Digital: http://burdon.zonalibre.org/archives/2005_01.html

- Carlino, P. (2005). Escribir, leer y aprender en la universidad. Buenos Aires: Fondo de Cultura Económica. 
- Chrobak, R. (1998). Metodologías para lograr aprendizaje significativo. Neuquén: Educo.

- Egipto: logros de ayer-retos de hoy. (s.f.). Recuperado 24/05/09 de http://cleoppatra. com/category/libro-de-los-muertos/

- Hispánica. (s.f.). Recuperado el 01 de Agosto de 2007 de http://poesiadelmomento.com/ hispanica/01mapa.html

- Huerta, P. (1998). La entrevista clínica y los mapas conceptuales. $2^{\circ}$ Simposio Nacional de la SEIEM. Pamplona, pp. 56-66.

- Johnson, D. W., Johnson, R. T., Holubec, E. J. (2004). El aprendizaje cooperativo en el aula. Buenos Aires: Paidós.

- Las Meninas. (s.f.). Recuperado el 01 de Agosto de 2007 de, http://es.wikipedia.org/ wiki/Las_Meninas

- Leiter. (2008). Diez pinturas inolvidables V (Rijksmuseum de Amsterdam). Recuperado 01 de Agosto de 2009, del sitio web de Escritura Digital: http://leiter.wordpress.com/tag/ rijksmuseum-amsterdam/

- $\quad$ Muñiz, W. (2010). Entre Doña Bárbara y la Gioconda: Un estudio paratextual sobre la novela cumbre de Rómulo Gallegos. Recuperado 20 de Diciembre de 2010, del sitio web de Escritura Digital: http://www.ucm.es/info/especulo/numero44/dbarbar.html

- Novak, J. y Gowin, D. (1988). Aprendiendo a aprender. Barcelona: Martínez Roca.

- $\quad$ Palou de Maté, C. (2003). Evaluar para enseñar y evaluar para acreditar. Palou de Maté (Comp.). La enseñanza y la evaluación. Una propuesta para matemática y lengua. (pp. 19-48) Buenos Aires: GEEMA - U.N.Co. Facultad de Cs. de la Educación - C.E.Di.Co.

- $\quad$ Pirámides de Egipto. (s.f.). Recuperado el 24 de Mayo de 2009 de http://www.piramidesdeegipto.com/enigmas/quien-creo-la-piramide-de-keops

- Rodríguez Conde, J. (2011). Aplicación de las Tic a la evaluación de alumnos universitarios. Recuperado el 01 Septiembre de 2011 del sitio web de Escritura Digital: http://campus.usal.es/ teoriaeducacion/rev_numero_06_2/n6_02_art_rodriguez_conde. htm[09/03/2011 10:36:36]

- Románico en Cuenca. (s.f.). Recuperado 01 de Agosto de 2009, de http://www.arteguias. com/romanico_cuenca.htm 